\title{
An Improved Design of Pressure Relay Based on Mechanical Pressure Gauge
}

\author{
Shan Chen ${ }^{1,{ }^{*}}$, Junchun $\mathrm{Ma}^{1}$, Yongbao Feng ${ }^{1}$, Changlin $\mathrm{Ma}^{1}$, Guanglei $\mathrm{Fu}^{1}$ \\ ${ }^{1}$ Research Inst. of High-tech Hongqing Town, Xi'an, Shanxi, China \\ *chenshan1223@126.com
}

Keywords: Mechanical Pressure Gauge, Pressure Relay, Improved Design.

\begin{abstract}
In order to involve problems of the pressure relay when using, this paper proposed an improved design of pressure relay based on mechanical pressure gauge. Firstly, the structure of the pressure relay and the existed problems when using were analyzed. Secondly, based on the mechanical pressure gauge's work principle and its structure characteristic, combining the deflection angle of the pointer with the threshold value of the pressure relay, this paper improved the pressure relay's structure, electrical circuit and control circuit. Experimental results showed that when using the pressure relay based on mechanical pressure gauge, its switch value was better than the original pressure relay, and the set point deviation was also significantly reduced. The design of pressure relay based on mechanical pressure gauge was feasible.
\end{abstract}

\section{Introduction}

For the hydraulic equipment, in order to achieve automatic control using the pressure as control signal, the pressure relay is one of the important components. It is an electro-hydraulic control element, which converts the pressure signal of oil into electric signal. However, the original pressure relay has many problems such as the switching value is too large, the set point deviation is too big, and the repetitive precision is too low and so on, which affect the reliability and safety of the hydraulic system [1-3].

In the process of technical measurement, because mechanical pressure gauges' elastic sensitive components have the high mechanical strength and the convenience of production, which make the mechanical pressure gauges have been widely used. But mechanical pressure gauges cannot convert pressure signals into electrical signal outputs.

Therefore, based on the working principle of the pressure relay, this paper reconstructed the mechanical pressure gauges, and implemented an improved pressure relay, which can not only accurately read the pressure value, and can reliably convert pressure values through electrical components into the control signal.

\section{The Structure Principle of Pressure Relay and Analysis of malfunction}

In the hydraulic system, through converting the oil pressure signal into electrical signals, the pressure relay can control the electromagnet, relays and other components, so that the oil circuit can perform the specified sequence of actions, and can also make the system stop working to protect the system security. The pressure relay is mainly consisting of the pressure - displacement conversion mechanism, the electrical micro-switches and so on [4-5].

When a plunger pressure relay working, the oil enters from the following oil inlet port, when the oil pressure reaches the set pressure of the pressure relay, under the function of the oil pressure, the plunger will move up and drive the lever, then switch will actions. The size of the pressure relay's set pressure can be changed by the compression spring, which structure is relatively simple, more common, but it has low sensitivity and low reliability of the action [6]. 
The malfunction of the pressure relay can be summarized as the switching value is too large, the set point deviation is too big, the repetitive precision is too low and so on, and its malfunctions are mainly the following reasons:

(1) Plunger stuck causes the pressure relay cannot work properly.

(2)For the film-type pressure relay, if the film is ruptured, the pressure oil will be leaked, which causes the pressure relay to appear abnormal phenomenon, and result in malfunction.

(3)By the external force, micro-switch generates position deviation, which causes malfunction.

(4)If there are too much dust on the micro-switch reed contact, which causes the micro-switch does not work and lead to malfunction.

(5)Due to technical problems in the manufacturing process, this leads to the failure of the pressure relay.

\section{Improved design of pressure relay based on mechanical pressure gauge}

Based on atmospheric pressure, pressure gauge is an instrument for the relative value of atmospheric pressure; it can also be used to measure the pressure of the fluid (gas, liquid). According to different sensitive components, the pressure gauge can be divided into some types, such as spring tube, diaphragm, bellows and so on.

Take the spring tube as an example to analyze the pressure gauge's performance and working principle, which is the most commonly used, and Fig. 1 shows its structure.

When the pressure oil entering into the spring tube, the tube end will be deformed, and the connecting rod will drive transmission mechanism to swing, which makes the pointer deflection, the pressure value can be read from the dial.

For a mechanical pressure gauge, the system pressure value is indicated through the pointer deflection, if the mechanical pressure gauge's deflection angle of the pointer can be combined with the threshold value of the pressure relay, when the system pressure value achieving the threshold value, the signal will be output.

Based on this, this paper designed the electrical circuit of a pressure gauge, as shown in Fig. 2.

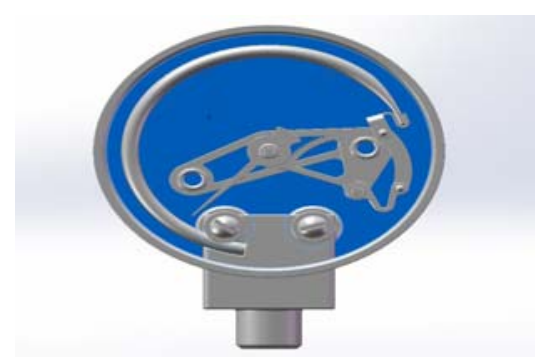

Figure 1. Structure diagram of the spring tube

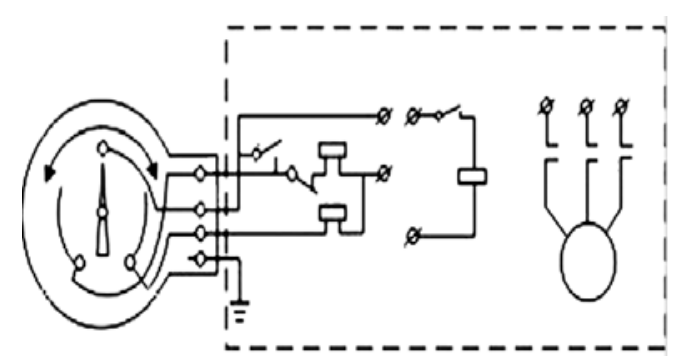

Figure 2. The electrical circuit

Its working principle: In the concrete operation process, firstly, set the upper pressure value and the lower pressure value by the rotary button on the outer side of the glass cover, the upper pressure value is the pressure value to be alarmed, and the lower value is the required pressure value; secondly, when the pressure of the measured medium enters into the spring tube, due to the emergence of new pressure, the spring tube will produce displacement, which will be amplified by the gear transmission mechanism, so, when the pointer on the dial indicating the pressure value, the moving contact will be driven to move at the same time, and when the pressure value exceeds the set lower pressure value, the pressure gauge will disconnect the normally closed switch, and the indicating lamp will bright. With the pressure rising, when the pressure value exceeds the set upper pressure value, the pressure gauge will disconnect the normally open switch, and the indicating lamp will bright, which prompts the pressure surpasses the upper value, must take the corresponding measures to reduce the pressure. At the same time, contacting with the set pointer (upper value or lower value) can cause the circuit be disconnected or connected, which achieve the purpose of automatic control and alarm. 
How to ensure the active contact and the pointer link reliably, it needs to improve the traditional structure of the pressure gauge.

Fig. 3 shows the improved structure of the pressure gauge, based on the mechanical pressure gauge, it adds a set pointer (as shown in Fig. 4) and a follower pointer (as shown in Fig. 5), at the same time, the original indicator pointer has been improved, which adds a blocking contact, as shown in Fig. 6 . The relationship between them is shown in Fig. 7.

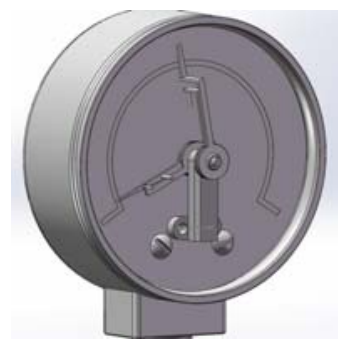

Figure 3. Improved structure of the pressure gauge

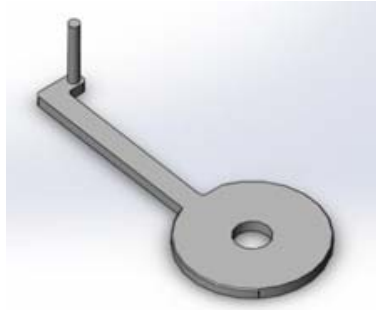

Figure 5. Follower pointer

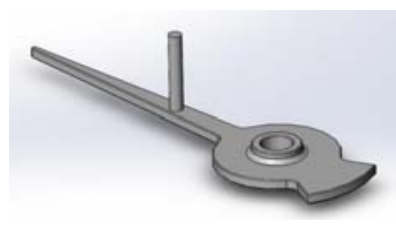

Figure 6. Improved pointer

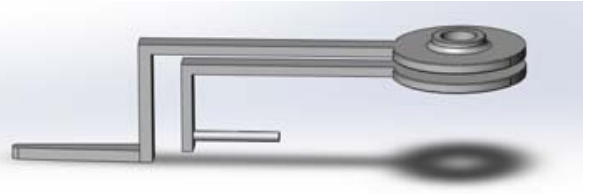

Figure 4. Set pointer

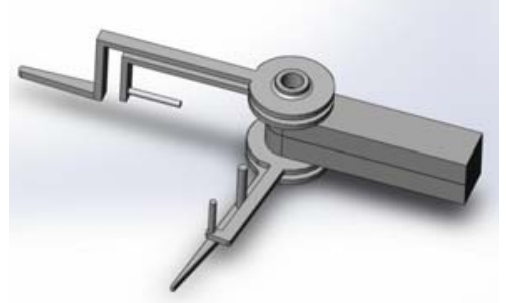

Figure 7. Relationship between pointers

In the follower pointer, there is a small spring, which provides pre-tightening force, impacting the follower pointer on the improved indicator pointer, and it will be held up by column on the indicator pointer, so, when the pressure rising, it will rotate with the improved pointer together.

When they rotating to the set point value of the set pointer, the follower pointer's magnetic contact will contact with the set pointer's magnetic contact, and the circuit is turned on, the follower pointer stops rotating, under the magnetic force, the two magnetic contacts will not contact poorly, although the pressure fluctuates, and the indicator pointer will continue to rotate in the scope of the range with the pressure increasing. When the measured pressure decreases, the indicator pointer will rotate reversely, and when reaches the set point value, the column on the indicator pointer will re-contact with the follower pointer, with the pressure continues to decrease, the indicator pointer will drive the follower pointer to reverse, so that the follower pointer's magnetic contact will disengage from the set pointer's magnetic contact, and the circuit is turned off. Therefore, the function of the pressure relay is realized, and at the same time, its malfunction is overcome.

\section{Experimental results and analysis}

In order to verify whether the sensitivity to the voltage change of the improved pressure relay was improved the experiment of testing to upper and lower switching values of the pressure relay was made in this paper. The experimental results were shown in Table.1 and Table.2. In the experiment, the type of PF-B8H4 pressure relay was used. The test environment was as follows. The test tool was pressure gauge with the precision of 0.25 , the working medium was hydraulic oil with N10, the temperature was $50^{\circ} \mathrm{C} \pm 2^{\circ} \mathrm{C}$.

From the experimental results, it can be concluded that when using the mechanical pressure gauge, its switching value is better than the original pressure relay, and the set point deviation is also significantly reduced. So the design of pressure relay based on mechanical pressure gauge this paper purposed is feasible. 
Table 1. Experimental results of the original pressure relay

\begin{tabular}{|l|l|l|l|l|}
\hline $\begin{array}{l}\text { The Set Point } \\
\text { Value Mpa }\end{array}$ & \multicolumn{2}{|l|}{$\mathbf{l}$} & \multicolumn{2}{|l|}{} \\
\hline $\begin{array}{l}\text { The Upper } \\
\text { Switching }\end{array}$ & 16.63 & 16.58 & 13.73 & 13.26 \\
\cline { 2 - 5 } \begin{tabular}{l} 
Value Mpa \\
\cline { 2 - 5 }
\end{tabular} & 16.20 & 16.02 & 13.23 & 13.15 \\
\hline $\begin{array}{l}\text { Average Value } \\
\text { Mpa }\end{array}$ & 16.30 & 16.48 & 13.06 & 13.13 \\
\hline Repeatability\% & 1.6 & 16.36 & 13.34 & 13.18 \\
\hline $\begin{array}{l}\text { The Lower } \\
\text { Switching } \\
\text { Value Mpa }\end{array}$ & 17.17 & 17.02 & 1.9 & 0.37 \\
\cline { 2 - 5 } & 17.45 & 17.18 & 14.06 & 14.36 \\
\hline $\begin{array}{l}\text { Average Value } \\
\text { Mpa }\end{array}$ & 17.64 & 17.52 & 14.73 & 14.39 \\
\hline Repeatability \% & 1.3 & 17.24 & 14.31 & 14.26 \\
\hline \multirow{2}{*}{$\begin{array}{l}\text { Deviation of } \\
\text { The Set \% }\end{array}$} & $\begin{array}{l}\text { The Upper Switching Value } \\
\text { Of Set Point Control }\end{array}$ & $\begin{array}{l}\text { The Lower Switching Value Of } \\
\text { Set Point Control }\end{array}$ \\
\cline { 2 - 5 } & 1.2 & 0.68 & 0.59 & 0.55 \\
\hline
\end{tabular}

Table 2. Experimental results of the mechanical pressure gauge

\begin{tabular}{|c|c|c|c|c|}
\hline $\begin{array}{l}\text { The Set Point } \\
\text { Value Mpa }\end{array}$ & \multicolumn{2}{|l|}{17} & \multicolumn{2}{|l|}{14} \\
\hline \multirow{3}{*}{$\begin{array}{l}\text { The Upper } \\
\text { Switching } \\
\text { Value Mpa }\end{array}$} & 16.73 & 16.78 & 13.85 & 13.64 \\
\hline & 16.50 & 16.62 & 13.33 & 13.57 \\
\hline & 16.77 & 16.68 & 13.65 & 13.35 \\
\hline $\begin{array}{l}\text { Average Value } \\
\text { Mpa }\end{array}$ & 16.67 & 16.69 & 13.61 & 13.52 \\
\hline Repeatability\% & 0.77 & 0.46 & 1.48 & 0.82 \\
\hline \multirow{3}{*}{$\begin{array}{l}\text { The Lower } \\
\text { Switching } \\
\text { Value Mpa }\end{array}$} & 17.05 & 17.08 & 14.08 & 14.04 \\
\hline & 17.30 & 17.27 & 14.12 & 14.21 \\
\hline & 17.44 & 17.42 & 14.54 & 14.32 \\
\hline $\begin{array}{l}\text { Average Value } \\
\text { Mpa }\end{array}$ & 17.26 & 17.25 & 14.25 & 14.19 \\
\hline Repeatability \% & 1.11 & 0.97 & 1.31 & 0.8 \\
\hline \multirow{2}{*}{$\begin{array}{l}\text { Deviation of } \\
\text { The Set } \%\end{array}$} & \multicolumn{2}{|c|}{$\begin{array}{l}\text { The Upper Switching Value } \\
\text { Of Set Point Control }\end{array}$} & \multicolumn{2}{|c|}{$\begin{array}{l}\text { The Lower Switching Value Of } \\
\text { Set Point Control }\end{array}$} \\
\hline & 0.75 & 0.73 & 0.70 & 0.54 \\
\hline
\end{tabular}

\section{Summary}

This paper firstly analyzes the working principle and structural characteristics of the mechanical pressure gauge, secondly, improves pressure gauge, and puts forward an improved design scheme of the pressure relay based on the photoelectric switch. Compared with the traditional pressure relay, it can be concluded that the improved pressure relay can not only overcome the problems existing in the traditional pressure relay, but also reduce the set point deviation and improve the measurement accuracy greatly.

\section{References}

[1] LIU Bao-jie, YANG Qing-wen, WU Xiang. Current Research and Development Trend of Fault Diagnosis Technology for Hydraulic System [J]. Hydraulics Pneumatics \& Seals, 2016, 08: 68-71.

[2] WU Yuan-hong. Discussion of Hydraulic Troubleshooting Phenomena Inverse Inference [J]. Hydraulics Pneumatics \& Seals, 2013, 01: 61-64. 
[3] ZHANG Li. Evaluation of Vncertainty about Indication Error of Electric Contact Filled System Thermoneter in Result of Measurement [J]. Metrology \& Measurement Technique,2009, 36(5): 88-89.

[4] Li Chunzhi. Design of Pressure Relay [J]. Hydraulics Pneumatics \& Seals, 2003, 01: 12-15.

[5] XU Yi1, YANG Zhengrui, WANG Yinghong, WEN Jiquan. Computer- aided Test \& Control System of Hydraulic Pressure- Compensatory and Pressure- Relay [J]. Hydromechatronics Engineering, 2004, 04: 161-162.

[6] ZHAO Xianqiong, LIU Yilun, YANG Xiaohong, HU Suyun. Research on Pressure Relay of New Type with Dif ferential Pressure [J]. Journal of Changsha Communications University, 2002, 18(1): 18-21. 